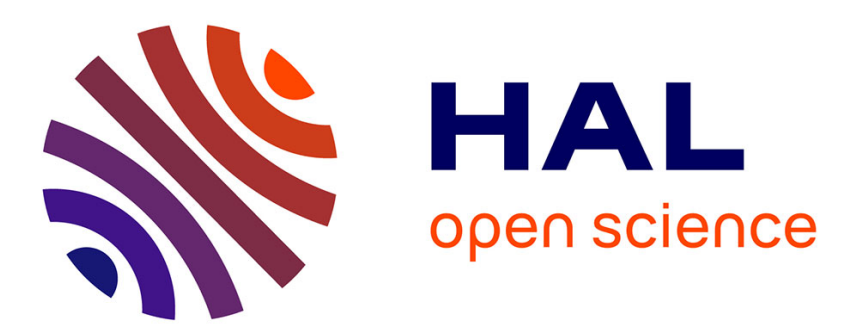

\title{
Necessary conditions for the generation of acoustic solitons in magnetospheric and space plasmas with hot ions
}

\author{
F. Verheest, M. A. Hellberg, G. S. Lakhina
}

\section{- To cite this version:}

F. Verheest, M. A. Hellberg, G. S. Lakhina. Necessary conditions for the generation of acoustic solitons in magnetospheric and space plasmas with hot ions. Astrophysics and Space Sciences Transactions, 2007, 3 (1), pp.15-20. hal-00297485

\section{HAL Id: hal-00297485 \\ https://hal.science/hal-00297485}

Submitted on 7 Sep 2007

HAL is a multi-disciplinary open access archive for the deposit and dissemination of scientific research documents, whether they are published or not. The documents may come from teaching and research institutions in France or abroad, or from public or private research centers.
L'archive ouverte pluridisciplinaire HAL, est destinée au dépôt et à la diffusion de documents scientifiques de niveau recherche, publiés ou non, émanant des établissements d'enseignement et de recherche français ou étrangers, des laboratoires publics ou privés. 


\title{
Necessary conditions for the generation of acoustic solitons in magnetospheric and space plasmas with hot ions
}

\author{
F. Verheest ${ }^{1,2}$, M. A. Hellberg ${ }^{2}$, and G. S. Lakhina ${ }^{3}$ \\ ${ }^{1}$ Sterrenkundig Observatorium, Universiteit Gent, Krijgslaan 281, B-9000 Gent, Belgium \\ ${ }^{2}$ School of Physics, University of KwaZulu-Natal, Private Bag X54001, Durban 4000, South Africa \\ ${ }^{3}$ Indian Institute of Geomagnetism, New Panvel (West), Navi Mumbai 410 218, India
}

Received: 25 June 2007 - Revised: 16 August 2007 - Accepted: 16 August 2007 - Published: 7 September 2007

\begin{abstract}
Necessary conditions are discussed for the possible generation of large solitary acoustic modes in plasmas with one or more ion species which are hotter than some or all of the electron species. The analysis is based on a fluid dynamic approach. It is found that in most of these configurations the existence ranges for the solitary wave velocities are very narrow and close to one of the thermal velocities. In the latter situation, linear Landau damping may prevent the generation of nonlinear structures. The analysis indicates that both inertial and thermal effects for the ions need to be kept in the description, thus rendering an analytical investigation much more intricate.
\end{abstract}

\section{Introduction}

Solitary waves (in the form of bipolar pulses in the electric field parallel to the background magnetic field) have been identified throughout the Earth's magnetosphere at narrow boundaries, such as the plasma sheet boundary layer (Matsumoto et al., 1994; Frantz et al., 1998), the magnetosheath (Pickett et al., 2003), the bow shock (Bale et al., 1998) and in strong currents, such as those associated with the auroral acceleration region (Bounds et al., 1999). Except in the ion beam regions of the auroral zone, these solitary waves are reputed to be positive electrostatic potential structures, and also presumed to be associated with high-frequency disturbances.

Earlier theoretical models based on solitary electronacoustic structures in two-electron-temperature plasmas could explain observations of waves, provided that these had negative electrostatic potentials (Mace et al., 1991; Dubouloz et al., 1991). To explain positive potential structures, extensions to the models have been made by including an electron beam in what are then in effect three-electron-component models (Berthomier et al., 2000; Mace and Hellberg, 2001).

Correspondence to: F. Verheest (Frank.Verheest@UGent.be)
It is shown that below some critical electron beam velocity the nonlinear structures can have a positive potential signature.

More recently, the possibility to obtain positive potential electron-acoustic solitons in two-electron-temperature plasmas was pointed out, provided hot electron inertia is retained (Cattaert et al., 2005; Verheest et al., 2005). Although the latter model obviates the need for an electron beam component, it requires a rather high density of cooler electrons, which does not seem to correspond to the observations. However, it should be noted that at present the instrument resolution is too poor to determine cold electron distributions accurately.

On the other hand, nonlinear solitary structures have been observed in the plasma sheet boundary layer regions (Parks et al., 1984; Onsager et al., 1993), which may play a key role in supporting parallel electric fields in the Earth's magnetotail. The observations indicate the existence of background electrons, cold electron beams, background cool ions and warm ions or ion beams. This has motivated studies of models where some of the ions might be hotter than the electrons (e.g. Tagare et al., 2004; Kakad et al., 2007).

In this paper we will illustrate that great care has to be taken in the use of the terminology "hot" and "cold", and in treating the ions as inertialess species (i.e., using Boltzmann density distributions for the ions). After this Introduction, we recall in Sect. 2 some necessary conditions for the generation of solitary acoustic waves in multispecies plasmas, and apply these in Sect. 3 to hydrogen plasmas, in order to contrast well known facets of plasmas having cool ions and hot electrons with the converse case where ions are hotter than electrons. Section 4 focuses on several possibilities in twoelectron-temperature plasmas, including a case where the hot ions have thermal velocities that are intermediate between those of the cool and the hot electrons. Section 5 is devoted to the more unusual two-ion-temperature plasma models, where both ion species are hotter than the electrons. Finally, Sect. 6 contains our conclusions, indicating that hotter ions imply

Published by Copernicus Publications on behalf of the Arbeitsgemeinschaft Extraterrestrische Forschung e.V. 
severely circumscribed and very narrow existence ranges for solitons.

\section{Necessary existence conditions for acoustic modes in multispecies plasmas}

We will treat electrostatic solitons in unmagnetized plasmas, or, when the plasmas are magnetized, at propagation parallel to the static magnetic field. In common with many other authors, we use "soliton" as shorthand for solitary localized nonlinear pulses or dips in the electrostatic potential, and describe them in a frame in which they appear stationary. Details of the formalism can be found e.g. in overview type papers by McKenzie et al. (2004) or Verheest et al. (2004) and in references contained therein.

To follow our exposition, we briefly recall some elements of the method. In a frame co-moving with the nonlinear structure, the fluid equations of continuity and momentum can easily be integrated, with vanishing boundary conditions far away from the soliton. Since the modes are electrostatic, there are no wave magnetic effects and of Maxwell's equations only Poisson's equation remains to be considered. Using the species' mass flux and momentum invariants, this can formally be integrated to obtain a mechanical energy-type integral for the electrostatic potential $\varphi$, of the generic form (McKenzie et al., 2004; Verheest et al., 2004)

$\frac{1}{2}\left(\frac{d \varphi}{d x}\right)^{2}=S(\varphi, V)$

where $V$ is the speed of the solitary wave in an inertial frame. Undoing one integration with respect to the space coordinate $x$ gives

$\frac{d^{2} \varphi}{d x^{2}}=\frac{d S}{d \varphi}(\varphi, V)$

Because of the undisturbed conditions far away, the integration constants have been taken such that

$S(0, V)=0, \quad \frac{d S}{d \varphi}(0, V)=0$,

ensuring that $S(\varphi, V)$ has a double root at $\varphi=0$.

In order to have a maximum or minimum value of the electrostatic potential $\varphi_{m} \neq 0$ in the center of the structure, we need to find roots of $S\left(\varphi_{m}, V\right)=0$ outside $\varphi=0$. A prerequisite (but not a sufficient condition!) for that to happen is that $S(\varphi, V)$ has the proper convexity at $\varphi=0$, namely

$\frac{d^{2} S}{d \varphi^{2}}(0, V)>0$.

If one goes through the algebra, this necessary convexity condition is (McKenzie et al., 2004; Verheest et al., 2004)

$F(V):=\sum_{\alpha} \frac{\omega_{p \alpha}^{2}}{c_{t \alpha}^{2}-V^{2}}>0$.

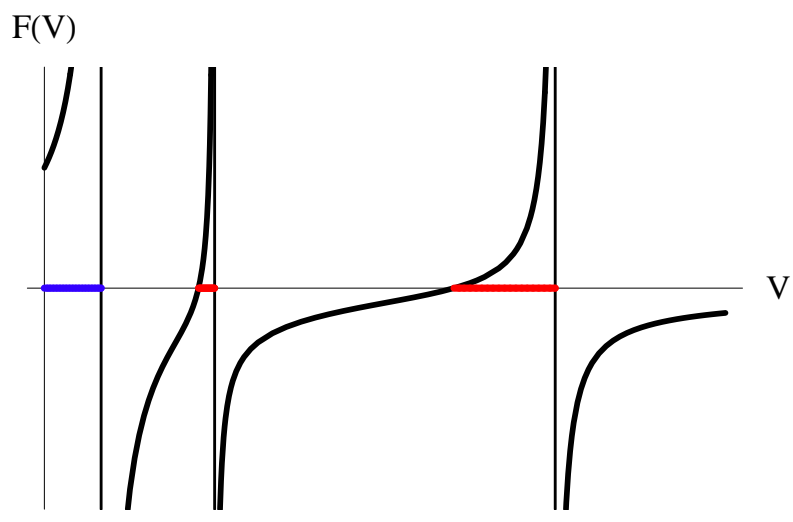

Fig. 1. Generic graph for the existence domains in a threecomponent plasma. The red ranges yield acceptable necessary conditions, the blue one not. The precise labeling of the asymptotes depends on the ordering of the different thermal velocities.

Here $\omega_{p \alpha}$ and $c_{t \alpha}$ refer to the plasma frequency and the generalized thermal velocity of the plasma species $\alpha$, respectively. It is worth stressing that these conditions are only necessary! The sufficient conditions, unfortunately, require the determination of the possible roots $\varphi_{m}$ of $S(\varphi, V)$, which often is not amenable to a complete analytical discussion (McKenzie et al., 2004; Verheest et al., 2004). In what follows, we will focus on the ranges allowed by Eq. (5), and leave the full discussion of explicit examples or plasma compositions for further work.

From the requirement (5) we find one or more critical acoustic velocities $V_{a}$, being defined as the roots of $F(V)=0$, and lying between two successive thermal velocities. We further know from fluid dynamic considerations that at least one species needs to be supersonic ("cold", in the sense that its thermal velocity is smaller than $V$ ) and at least one subsonic ("hot", with a thermal velocity larger than $V$ ) (McKenzie et al., 2004; Verheest et al., 2004). Note that hot and cold are expressed purely in terms of thermal velocities compared to the structure velocity, and do not immediately refer to temperatures.

This leads to generic graphs where the necessary existence domains for stationary solitary structures require that $V_{a}<V$, before the next asymptote at a thermal velocity is encountered. As illustrated in Fig. 1, in a three-component plasma there are two acoustic existence ranges for solitary waves, indicated in red, whereas the lowest possible range indicated in blue does not fit the bill, all three species then being subsonic. With more species one gets more acoustic ranges.

As an aside, in the domains where $F(V)=-k^{2} \leq 0$ one recovers linearized harmonic waves with frequency $\omega$ and wave number $k$, provided $V$ is interpreted as the linear phase velocity $\omega / k$. Were one to consider a plasma with cool ions, cool and hot electrons, the successive ranges would refer to 
linear ion-acoustic, electron-acoustic and Langmuir waves, respectively, at increasing $V$.

\section{Discussion of acoustic modes in hydrogen plasmas}

In order to clarify potential difficulties with plasma models where some or all of the ions might be hotter than the electrons, we have to recall well-known elements of simpler or more usual plasma compositions. We start from the soliton condition for a hydrogen plasma,

$F(V):=\frac{\omega_{p e}^{2}}{c_{t e}^{2}-V^{2}}+\frac{\omega_{p i}^{2}}{c_{t i}^{2}-V^{2}}>0$,

which yields an acoustic velocity defined through

$V_{a}^{2}=\frac{m_{i} c_{t i}^{2}+m_{e} c_{t e}^{2}}{m_{i}+m_{e}}$.

Hence $V$ has to obey

$\min \left\{c_{t e}^{2}, c_{t i}^{2}\right\}<V_{a}^{2}<V^{2}<\max \left\{c_{t e}^{2}, c_{t i}^{2}\right\}$,

but we have not ordered the thermal velocities yet, for which two cases are possible.

The first one, $c_{t i}<c_{t e}$, seems the more applicable one, and for nonlinear acoustic modes in this ordering we need $c_{t i}<V_{a}<V<c_{t e}$. Written in terms of generalized kinetic temperatures $T_{e}=m_{e} c_{t e}^{2}$ and $T_{i}=m_{i} c_{t i}^{2}$ we get

$c_{t i}^{2}<V_{a}^{2}=\frac{T_{i}+T_{e}}{m_{i}+m_{e}}<V^{2}<c_{t e}^{2}$.

When $T_{i}$ and $T_{e}$ are similar, the linear counterparts of the solitary waves studied here are heavily ion Landau damped, and so it is not obvious that nonlinear modes can be generated. Hence we take $T_{i} \ll T_{e}$, which is, however, more stringent than required by the soliton condition (5) alone. Since $m_{e} \ll m_{i}$ one arrives at the usual definition $V_{a}^{2} \simeq T_{e} / m_{i}$, so that indeed $c_{t i}^{2} \ll V_{a}^{2} \ll c_{t e}^{2}$. In this case a possible range is $V_{a}^{2}<V^{2} \ll c_{t e}^{2}$, but we can only study solitons that are slightly superacoustic.

The large separation between the characteristic speeds indicates that we could equally well have started with cold ions $\left(c_{t i} \ll V\right)$ and inertialess (Boltzmann) electrons $\left(V \ll c_{t e}\right)$ and arrived at a reasonable approximation to the full $V_{a}$. We recall that in a fluid model Boltzmann density distributions are obtained for isothermal species when inertial effects are omitted. The balance between thermal and electrostatic forces in the corresponding momentum equation then gives typical density distributions, e.g. for electrons, of the form $n_{e}=n_{e 0} \exp \left(e \varphi / T_{e}\right)$, where $n_{e}$ is the electron number density (with equilibrium value $n_{e 0}$ ) and $e$ is the unit charge in absolute value.

Could one have the ordering $c_{t i}<c_{t e}$, but with $T_{e}<T_{i}$ ? In order to avoid heavy Landau damping of the linear modes, one would need to have a sufficiently large window between the two thermal speeds for the phase speed to be clearly separated from them, i.e. $T_{i} / m_{i} \ll T_{e} / m_{e}$. However, substitution into Eq. (7) then leads to $V_{a}^{2} \simeq T_{i} / m_{i}$, and the linear wave is still subject to strong ion Landau damping.

The other, theoretically possible ordering might be that $c_{t e}<c_{t i}$ or $T_{e} / m_{e}<T_{i} / m_{i}$, so that $c_{t e}<V_{a}<V<c_{t i}$ can hold, but with what would appear to be unrealistically small electron temperatures, since $T_{e}<\left(m_{e} / m_{i}\right) T_{i} \ll T_{i}$. Putting $c_{t e}^{2}=c_{t i}^{2} \delta$, where $0 \leq \delta<1$, gives, with a return to the original definition (7), that

$V_{a}^{2}=\frac{m_{i}+m_{e} \delta}{m_{i}+m_{e}} c_{t i}^{2}<V^{2}<c_{t i}^{2}$.

The available window for $V$ is so narrow and $V_{a}$ so close to $c_{t i}$ that not only is heavy ion Landau damping expected, but in addition the ions can certainly not be treated as Boltzmann distributed (i.e. inertialess), for which $V \ll c_{t i}$ would be needed (Mace and Hellberg, 1993; Rice et al., 1993). All in all this is not a physically realistic regime. The underlying reason is that in this model the hot species is now heavy and the cold species is light: compared to the original ionacoustic model, one has swapped the role of the temperatures, but this cannot be done for the masses!

\section{Plasmas with two electron temperatures}

We now go to a more complicated but well-studied model, that of two-electron-temperature plasmas, for which the necessary condition for the existence of solitary structures is that

$\frac{\mu}{c_{t i}^{2}-V^{2}}+\frac{f}{c_{t e c}^{2}-V^{2}}+\frac{1-f}{c_{t e h}^{2}-V^{2}}>0$.

Here $f=n_{c e 0} / n_{i 0}<1$ is the fractional cool electron density, the mass ratio $\mu=m_{e} / m_{i}$ is obviously a small parameter and the cooler and hotter electron species have been subscripted by $e c$ and $e h$, respectively.

There are two possible acoustic regimes, and to discuss these we will first assume the usual ordering that $c_{t i}<c_{t e c}<c_{t e h}$. Taking in Eq. (11) brutally the limit $\mu \rightarrow 0$ gives

$V_{a H}^{2}=f c_{t e h}^{2}+(1-f) c_{t e c}^{2}$,

which corresponds to the (higher) electron-acoustic velocity in the model. At these high acoustic speeds, the ion dynamics hardly matter and they can be forgotten as an almost immobile background.

If we assume the stronger condition that $c_{t i}<c_{t e c} \ll V_{a H}<V<c_{t e h}$, we find a restriction on $f$, viz.

$1>\frac{V^{2}}{c_{\text {teh }}^{2}}>\frac{V_{a H}^{2}}{c_{\text {teh }}^{2}} \simeq f$.

If the hot electrons are treated as inertialess, then another strong inequality is needed, namely $V \ll c_{t e h}$, in order to neglect in their equation of motion the inertial effects in favour 
of the thermal contributions. An immediate consequence is the restriction to $f \ll 1$ and then only negative electrostatic potential solitons can be found (Mace et al., 1991). These are rarefactive for the hot electrons and the (cold) ions, but compressive for the cool electrons. As pointed out on various occasions, the labels rarefactive and compressive are ambiguous in multispecies plasma treatments.

In order to find in this two-electron-temperature model electron-acoustic solitons with a positive electrostatic potential, which are compressive for the hot electrons and the (cold) ions but rarefactive for the cool electrons, it is imperative to retain hot electron inertia in combination with the thermal effects (Cattaert et al., 2005; Verheest et al., 2005). The drawback of this model to generate positive electrostatic potential solitons is that a rather high fraction of cool electrons is needed, larger than can at present be inferred from available observations.

For ion-acoustic solitons in a two-electron-temperature model, we return to Eq. (11) and note that $\mu c_{t e c}^{2}=T_{e c} / m_{i}$ and likewise that $\mu c_{t e h}^{2}=T_{e h} / m_{i}$. Retaining these terms before taking the limit $\mu \rightarrow 0$ yields the (lower) ion-acoustic velocity,

$V_{a L}^{2}=c_{t i}^{2}+\frac{\mu c_{t e c}^{2} c_{t e h}^{2}}{f c_{t e h}^{2}+(1-f) c_{t e c}^{2}}$.

Introducing the effective electron temperature through

$\frac{1}{T_{\mathrm{eff}}}=\frac{f}{T_{e c}}+\frac{1-f}{T_{e h}}$

allows us to rewrite $V_{a L}^{2}$ as

$V_{a L}^{2}=\frac{T_{i}+T_{\mathrm{eff}}}{m_{i}}$.

For the nonlinear ion-acoustic modes, one has traditionally supposed that stronger inequalities hold, viz. $c_{t i} \ll V_{a L}<V \ll c_{t e c}<c_{t e h}$, allowing the approximations that the ions are cold and the electrons hot enough to be treated as inertialess.

Another possible ordering is the one used by $\mathrm{Yu}$ and Shukla (1983), with cold and hot electrons, in the presence of hot ions, in the sense that $0 \simeq c_{t e c}<c_{t i}<c_{t e h}$. Using now Eq. (11) with $c_{t e c} \simeq 0$ yields

$$
\begin{aligned}
& V_{a 1}^{2}=c_{t i}^{2}\left(1+\frac{c_{t e h}^{2}-c_{t i}^{2}}{c_{t i}^{2}-f c_{t e h}^{2}} \mu\right), \\
& V_{a 2}^{2}=f c_{t e h}^{2}\left(1+\frac{(1-f) c_{t e h}^{2}}{f c_{t e h}^{2}-c_{t i}^{2}} \mu\right),
\end{aligned}
$$

by expanding up to first order in $\mu$ and assuming that $f c_{t e h}^{2}-c_{t i}^{2}$ is not so small as to invalidate the expansion. The discussion of possible existence ranges hinges on the relative ordering of $f c_{t e h}^{2}$ and $c_{t i}^{2}$.

First, let us assume that $f$ is not too small, so that $c_{t i}^{2}<f c_{t e h}^{2}<c_{t e h}^{2}$. In that case the lower acoustic range is given by $V_{a 1}<V<c_{t i}$ and the higher one by $V_{a 2}<V<c_{t e h}$. The first range will be rather narrow and lead to ion Landau damping, while the second one will need to keep the window between $f$ and 1 sufficiently wide to be of interest. The treatment here will probably have to retain the full hot electron inertia, besides the thermal effects, much as was discussed earlier in this Section (Cattaert et al., 2005).

The other possibility is that $f$ is small enough so that $f c_{t e h}^{2}<c_{t i}^{2}<c_{t e h}^{2}$. Then the lower acoustic range is given by $V_{a 2}<V<c_{t i}$ and the higher one by $V_{a 1}<V<c_{t e h}$. To keep these ranges wide enough the gaps between $f c_{\text {teh }}^{2}$ and $c_{t i}^{2}$ and between $c_{t i}^{2}$ and $c_{t e h}^{2}$ should be not too small.

\section{Plasmas with two ion temperatures}

Having recalled some of the aspects of the two-electrontemperature models, we turn to the much less studied and harder to realize two-ion-temperature plasmas. The necessary condition for acoustic solitons now becomes

$\frac{1}{c_{t e}^{2}-V^{2}}+\frac{f \mu}{c_{t i c}^{2}-V^{2}}+\frac{(1-f) \mu}{c_{t i h}^{2}-V^{2}}>0$,

with a redefined fractional cool ion density $f=n_{c i 0} / n_{e 0}<1$, and with self-evident labels $i c$ and $i h$ for the cooler and hotter ion species, respectively. Here, the two possible regimes are

$$
\begin{aligned}
V_{a L}^{2} & =c_{t i c}^{2}+f \mu\left(c_{t e}^{2}-c_{t i c}^{2}\right), \\
V_{a H}^{2} & =c_{t i h}^{2}+(1-f) \mu\left(c_{t e}^{2}-c_{t i h}^{2}\right),
\end{aligned}
$$

up to first order in the small parameter $\mu$. Regardless of how one orders $c_{t e}$ with respect to $c_{t i c}<c_{t i h}$, it can be shown that $V_{a L}$ refers to the lower acoustic velocity and $V_{a H}$ to the higher acoustic velocity.

Starting the ordering of $c_{t e}$ from high up, one could have that $T_{e} \lesssim T_{i h}$, but this would still make $c_{t i h} \ll c_{t e}$. Hence, the electrons could almost be taken out of the description as being very hot (discussed in thermal velocities rather than temperatures), and then we can draw on earlier results (Hellberg et al., 2006). In that paper, a model involving hot and cool ions, "super-hot" electrons and massive, immobile dust grains was used to consider ion-acoustic-like solitons supported by ions satisfying $c_{t e} \gg c_{t i h} \gg V_{a} \gg c_{t i c} \gg c_{d}$, where the subscript $d$ refers to dust. However, it was found that within these approximations, the distribution of charge between electrons and negative dust grains was arbitrary, and hence one can ignore the dust grains completely. The result is that the ion-acoustic-like solitons in this regime are directly analogous to the electron-acoustic solitons found in Sect. 3, and an equivalent existence diagram in parameter space is obtained.

Lowering $T_{e}$ brings us to the range where $c_{t e}$ is comparable to $c_{t i h}$, and results are not expected to be significantly different from the special case that $c_{t e}=c_{t i h}$. 
Then we see that $V_{a H}=c_{t i h}$ and only $c_{t i c}<V_{a L}<c_{t i h}$ can define a meaningful acoustic regime. Note that since $V_{a L}^{2}=c_{t i c}^{2}+f \mu\left(c_{t i h}^{2}-c_{t i c}^{2}\right)$ is very close to but larger than $c_{t i c}^{2}$, a proper regime avoiding large Landau damping of the solitary structure by the cool ion component will need $V_{a L}^{2} \ll V^{2} \ll c_{t i h}^{2}$. This model might offer a more realistic approach for certain magnetospheric applications, but needs further study to ascertain the precise nonlinear character of the solitary waves.

The other obvious limit is that $c_{t e}=c_{t i c}$, although that would already require an extremely small electron temperature $T_{e}=\left(m_{e} / m_{i}\right) T_{i c}$. Then it follows from Eq. (19) that $V_{a L}=c_{t i c}$, and only $c_{t i c}<V_{a H}<c_{t i h}$ can define an acoustic regime. However, even though $V_{a H}^{2}=c_{t i h}^{2}-(1-f) \mu\left(c_{t i h}^{2}-c_{t i c}^{2}\right)$ is smaller than $c_{t i h}^{2}$, it is only marginally so. This leaves us with a tiny window for $V$ just below $c_{t i h}$, with heavy Landau damping by the hot ions. Coupled to the tiny electron temperature, this ordering does not seem very realistic.

Going even lower in $T_{e}$ is even more problematic, for reasons which have already been encountered in Sect. 2, dealing with $c_{t e}<c_{t i}$ in a hydrogen plasma. Hence, the use of Boltzmann distributions for the hot ions would seem to exceed the validity of the assumptions underpinning this approximation, although further work is needed to ascertain whether this is also the case in the presence of an electron beam.

\section{Conclusions}

In this paper we have discussed necessary conditions for the generation of large solitary acoustic modes in plasmas with one or more ion species which are hotter than some or all of the electron species. Indications are that in most of these configurations the existence ranges for the solitary wave velocities are severely circumscribed, very narrow and close to one of the thermal velocities. Not only will linear Landau damping prevent the generation of nonlinear structures, but the ions cannot be treated as inertialess. Quite to the contrary, both the inertial and thermal effects will have to be kept in the description, which renders an analytical investigation much more intricate.

In order to get this point across, we have found it helpful to contrast our present discussion with analogous considerations for large ion- and electron-acoustic modes in better known plasma models, where one or two electron constituents are hotter than the ions. Because of the additional complications in hot ion plasmas, we have not yet incorporated the influence of possible beam components, but will certainly do so in future work. Electron or ion beams might also help in overcoming linear Landau damping and have the possibility of generating larger structures.

Furthermore, we have neglected the effect of the magnetic field on the wave generation. This is justified for waves propagating strictly parallel to the static magnetic field. For oblique propagation, the effects of an external magnetic field can be important for linear as well as nonlinear waves, unless the linear wave frequencies are much higher than the gyrofrequencies of all the plasma species.

To conclude, full nonlinear analytical and numerical evaluations have to be undertaken to check out the different possibilities outside traditional ion- and electron-acoustic models. This is beyond the scope of this preliminary discussion.

Acknowledgements. This work was supported in part by a bilateral cooperation grant on the "Physics of Space Plasmas" between Flanders (Onderzoekfonds Universiteit Gent) and South Africa (National Research Foundation, Grant number 2068816). FV also thanks the Fonds voor Wetenschappelijk Onderzoek (Vlaanderen) for a research grant, MAH the National Research Foundation (Grant number FA2006041000033) and GSL the Council of Scientific and Industrial Research (India) for support under the Emeritus Scientist Scheme.

Edited by: H.-J. Fahr

Reviewed by: two referees

\section{References}

Bale, S. D., Kellogg, P. J., Larson, D. E., Lin, R. P., Goetz, K., and Lepping, R. P.: Bipolar electrostatic structures in the shock transition region: Evidence of electron phase space holes, Geophys. Res. Lett., 25, 2929-2932, 1998.

Berthomier, M., Pottelette, R., Malingre, M., and Khotyaintsev, Yu.: Electron-acoustic solitons in an electron-beam plasma system, Phys. Plasmas, 7, 2987-2994, 2000.

Bounds, S. R., Pfaff, R. F., Knowlton, S. F., Mozer, F. S., Temerin, M. A., and Kletzing, C. A.: Solitary potential structures associated with ion and electron beams near $1 \mathrm{RE}$ altitudes, J. Geophys. Res., 104, 28709-28718, 1999.

Cattaert, T., Verheest, F., and Hellberg, M. A.: Potential hill electron-acoustic solitons and double layers in plasmas with two electron species, Phys. Plasmas, 12, 042901, 2005.

Dubouloz, N., Pottelette, R., Malingre, M., and Treumann, R. A.: Generation of broadband electrostatic noise by electron acoustic solitons, Geophys. Res. Lett., 18, 155-158, 1991.

Franz, J. R., Kintner, P. M., and Pickett, J.S.: POLAR observations of coherent electric field structures, Geophys. Res. Lett., 25, 1277-1280, 1998.

Hellberg, M. A., Verheest, F., and Cattaert, T.: Existence domains for nonlinear structures in complex two-ion-temperature plasmas, J. Phys. A: Math. Gen., 39, 3137-3146, 2006.

Kakad, A. P., Singh, S. V., Reddy, R. V., Lakhina, G. S., Tagare, S. G., and Verheest, F.: Generation mechanism for electron acoustic solitary waves, Phys. Plasmas, 14, 052305 (2007).

Mace, R. L. and Hellberg, M. A.: Dust-acoustic double layers: Ion inertial effects, Planet. Space Sci., 41, 235-244, 1993.

Mace, R. L. and Hellberg, M. A.: The Korteweg-de VriesZakharov-Kuznetsov equation for electron-acoustic waves, Phys. Plasmas, 8, 2649-2656, 2001.

Mace, R. L., Baboolal, S., Bharuthram, R., and Hellberg, M. A.: Arbitrary-amplitude electron-acoustic solitons in a two-electroncomponent plasma, J. Plasma Phys., 45, 323-338, 1991. 
Matsumoto, H., Kojima, H., Miyatake, T., Omura, Y., Okada, M., Nagano, I., and Tsutsui M.: Electrostatic Solitary Waves (ESW) in the magnetotail: BEN wave forms observed by GEOTAIL, Geophys. Res. Lett., 21, 2915-2918, 1994.

McKenzie, J.F., Dubinin, E., Sauer, K., and Doyle, T.B.: The application of the constants of the motion to nonlinear stationary waves in complex plasmas: a unified fluid dynamic point of view, J. Plasma Phys., 70, 431-462 (2004).

Onsager, T. G., Thomsen, M. F., Elphic, R. C., Gosling, J. T., Anderson, R. R., and Kettmann, G.: Electron generation of electrostatic waves in the plasma sheet boundary layer, J. Geophys. Res., 98, 15509-15520, 1993.

Parks, G. K., McCarthy, M., Fitzenreiter, R. J., Etcheto, J., Anderson, K. A., Anderson, R. R., Eastman, T. E., Frank, L. A., Gurnett, D. A., Huang, C., Lin, R. P., Lui, A. T. Y., Ogilvie, K. W., Pedersen, A., Rème, H., and Williams, D. J.: Particle and field characteristics of the high latitude plasma sheet boundary layer, J. Geophys. Res., 89, 8885-8906, 1984.

Pickett, J. S., Menietti, J. D., Gurnett, D. A., Tsurutani, B., Kintner, P. M., Klatt, E., and Balogh, A.: Solitary potential structures observed in the magnetosheath by the Cluster spacecraft, Nonlin.
Proc. Geophys., 10, 3-11, 2003.

Rice, W. K. M., Hellberg, M. A., Mace, R. L., and Baboolal, S.: Finite electron mass effects on the existence of ion-acoustic solitons in a two electron temperature plasma, Phys. Lett. A, 174, 416-420, 1993.

Tagare, S. G., Singh, S. V., Reddy, R. V., and Lakhina, G. S.: Electron acoustic solitons in the Earth's magnetotail, Nonlin. Proc. Geophys., 11, 215-218, 2004.

Verheest, F., Cattaert, T., Lakhina, G. S., and Singh, S. V.: Gasdynamic description of electrostatic solitons, J. Plasma Phys., 70, 237-250, 2004.

Verheest, F., Cattaert, T., and Hellberg, M. A.: Compressive and rarefactive electron-acoustic solitons and double layers in space plasmas, Space Sci. Rev., 121, 299-311, 2005.

Yu, M. Y. and Shukla, P. K.: Linear and non-linear modified electron-acoustic waves, J. Plasma Phys., 29, 409-413, 1983. 